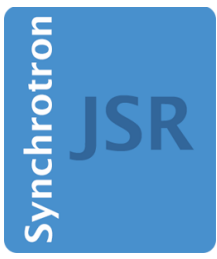

JOURNAL OF

SYNCHROTRON

RADIATION

Volume 22 (2015)

Supporting information for article:

Identifying and quantifying radiation damage at the atomic level Markus Gerstel, Charlotte M. Deane and Elspeth F. Garman 


\section{Identifying and quantifying radiation damage at the atomic level}

Gerstel, M., Deane, C. M. \& Garman, E. F. (2015).

J. Synchrotron Rad. 22, doi:10.1107/S1600577515002131.

Table S1 Isotropic atomic $B$-factors present in the PDB subset.

\begin{tabular}{crc}
\hline Atomic $B$-factor $\left[\AA^{2}\right]$ & Number of atoms & Percentage of atoms \\
$B<0$ & 3 & $<0.01 \%$ \\
$B=0$ & 4,610 & $0.05 \%$ \\
$0<B<80$ & $10,143,262$ & $99.65 \%$ \\
$B \geq 80$ & 31,338 & $0.31 \%$
\end{tabular}

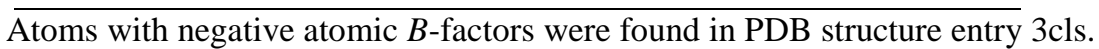

Table S2 Occupancy values present in the PDB subset.

\begin{tabular}{crc}
\hline Occupancy $o c c$ & Number of atoms & Percentage of atoms \\
$o c c<0$ & 5 & $<0.01 \%$ \\
$o c c=0$ & 9,177 & $0.09 \%$ \\
$0<o c c<0.5$ & 68,511 & $0.67 \%$ \\
$o c c=0.5$ & 234,192 & $2.30 \%$ \\
$0.5<o c c<1$ & 72,020 & $0.71 \%$ \\
$o c c=1$ & $9,795,303$ & $96.23 \%$ \\
$o c c>1$ & 5 & $<0.01 \%$
\end{tabular}

Some PDB structures contain occupancy values outside the valid probability range: the structure entries $1 \mathrm{ie} 0$ and 1 wul report occupancies above 1 , and the structure $3 \mathrm{hkw}$ reports occupancies below 0 . 
Table S3 Packing densities of protein secondary structure.

\begin{tabular}{|c|c|c|c|c|c|c|c|c|c|c|}
\hline $\begin{array}{l}\text { Density metric, } \\
\text { radius }\end{array}$ & $\begin{array}{l}\text { Secondary } \\
\text { structure }\end{array}$ & Min & Mean & Max & Variance & $\begin{array}{l}\text { Secondary } \\
\text { structure }\end{array}$ & Min & Mean & Max & Variance \\
\hline \multirow[t]{4}{*}{ Ooi, $8 \AA$} & $\mathrm{b}$ & 4 & 11.5 & 21 & 6.1 & $\mathrm{H}$ & 4 & 10.5 & 22 & 5.5 \\
\hline & $\mathrm{C}$ & 1 & 9.7 & 22 & 8.5 & I & 4 & 12.4 & 20 & 6.9 \\
\hline & E & 4 & 12.0 & 23 & 5.1 & $\mathrm{~T}$ & 8 & 9.6 & 22 & 8.1 \\
\hline & $\mathrm{G}$ & 4 & 9.5 & 21 & 7.1 & & & & & \\
\hline \multirow[t]{4}{*}{ Ooi, $13 \AA$} & b & 10 & 43.4 & 72 & 89.5 & $\mathrm{H}$ & 8 & 42.9 & 80 & 103.6 \\
\hline & $\mathrm{C}$ & 3 & 39.7 & 78 & 106.2 & I & 22 & 48.8 & 71 & 136.1 \\
\hline & $\mathrm{E}$ & 11 & 47.0 & 84 & 93.6 & $\mathrm{~T}$ & 5 & 38.6 & 82 & 106.8 \\
\hline & $\mathrm{G}$ & 8 & 39.6 & 75 & 104.2 & & & & & \\
\hline \multirow[t]{4}{*}{ Ooi, $14 \AA$} & $\mathrm{b}$ & 11 & 53.1 & 90 & 132.1 & $\mathrm{H}$ & 8 & 52.6 & 100 & 148.6 \\
\hline & $\mathrm{C}$ & 3 & 48.9 & 95 & 153.9 & I & 29 & 59.9 & 82 & 211.8 \\
\hline & $\mathrm{E}$ & 12 & 58.0 & 102 & 141.3 & $\mathrm{~T}$ & 5 & 47.8 & 96 & 154.3 \\
\hline & G & 9 & 49.1 & 96 & 150.4 & & & & & \\
\hline \multirow[t]{4}{*}{$\mathrm{ACN}, 8 \AA$} & $\mathrm{b}$ & 13 & 92.3 & 141 & 333.9 & $\mathrm{H}$ & 8 & 89.2 & 147 & 360.8 \\
\hline & $\mathrm{C}$ & 0 & 81.1 & 145 & 444.8 & I & 22 & 98.8 & 141 & 337.2 \\
\hline & E & 8 & 96.5 & 145 & 294.2 & $\mathrm{~T}$ & 8 & 79.9 & 144 & 447.6 \\
\hline & $\mathrm{G}$ & 8 & 82.2 & 141 & 424.6 & & & & & \\
\hline \multirow[t]{4}{*}{$\mathrm{ACN}, 13 \AA$} & $\mathrm{b}$ & 72 & 340.6 & 531 & $5,245.9$ & $\mathrm{H}$ & 46 & 336.2 & 542 & $5,757.5$ \\
\hline & $\mathrm{C}$ & 11 & 313.7 & 541 & $6,032.7$ & I & 158 & 385.7 & 517 & $7,705.1$ \\
\hline & E & 46 & 366.8 & 547 & $5,191.2$ & $\mathrm{~T}$ & 35 & 306.7 & 540 & $6,229.6$ \\
\hline & $\mathrm{G}$ & 48 & 314.5 & 539 & $6,074.3$ & & & & & \\
\hline \multirow[t]{4}{*}{$\mathrm{ACN}, 13.5 \AA$} & $\mathrm{b}$ & 74 & 376.7 & 589 & $6,416.0$ & $\mathrm{H}$ & 50 & 372.4 & 600 & $6,973.5$ \\
\hline & $\mathrm{C}$ & 14 & 348.1 & 597 & $7,293.6$ & I & 173 & 428.4 & 570 & $9,721.7$ \\
\hline & $\mathrm{E}$ & 53 & 405.5 & 601 & $6,377.9$ & $\mathrm{~T}$ & 37 & 340.8 & 597 & $7,541.5$ \\
\hline & G & 51 & 349.3 & 602 & $7,341.6$ & & & & & \\
\hline \multirow[t]{4}{*}{$\mathrm{ACN}, 14 \AA$} & $\mathrm{b}$ & 80 & 415.3 & 654 & $7,790.0$ & $\mathrm{H}$ & 52 & 410.9 & 666 & $8,366.2$ \\
\hline & $\mathrm{C}$ & 16 & 385.1 & 658 & $8,757.2$ & I & 190 & 474.0 & 633 & $12,043.5$ \\
\hline & $\mathrm{E}$ & 56 & 446.8 & 668 & $7,762.1$ & $\mathrm{~T}$ & 38 & 377.4 & 666 & $9,056.6$ \\
\hline & G & 57 & 386.4 & 659 & $8,809.8$ & & & & & \\
\hline
\end{tabular}

Range, mean and variance of different packing density metrics applied to the 2,704 selected PDB structures.

Both ACN and Ooi metrics are counts, so minimum and maximum values are integers. Mean and variance are given to $1 \mathrm{dp}$. The seven secondary structure labels are: alpha helix (H; $\mathrm{n}=3,323,956), 3-10$ helix (G; $n=428,747), \pi$-helix (I; $n=1,515)$, extended conformation ( $E ; n=2,351,831)$, isolated bridge ( $b ; n=120,856$ ), turn ( $\mathrm{T} ; \mathrm{n}=1,859,115)$ and coil $(\mathrm{C} ; \mathrm{n}=1,679,478)$ (Frishman \& Argos, 1995). 


\section{Table S4 List of the 2,704 selected PDB structures}

1A3A 1A3C 1A4I 1A7D 1AKY 1AL3 1ATZ 1B2L 1B2P 1B4V 1B4Z 1B5E 1B6A $1 \mathrm{~B} 80$ 1BQC 1BRT 1BW9 1BX4 1C7S 1CJW 1CQX 1CZF 1D2N 1D8W 1DBW 1DEO 1DGW 1DJ0 1DJE 1DK8 1DLW 1DPS 1DQG 1DQZ 1DWK 1DYP 1DYQ 1E19 1E2R 1E2W 1E30 1E42 1E4C 1E5M 1E6Y $1 \mathrm{E} 71$ 1E7R 1EDG 1EEX 1EJD 1ELK 1ELW 1EOK 1EQM 1EWF 1EYE

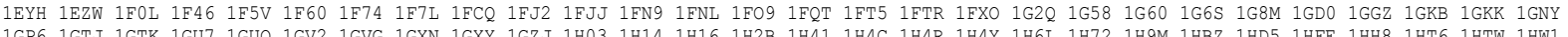

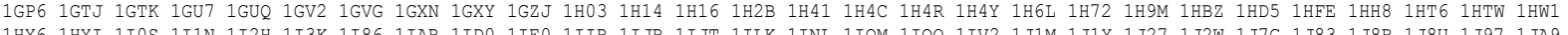

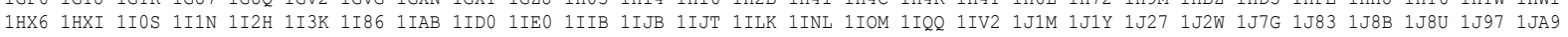

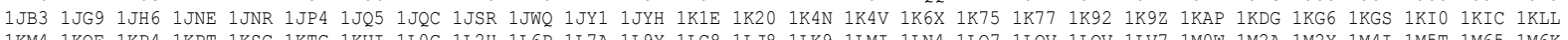

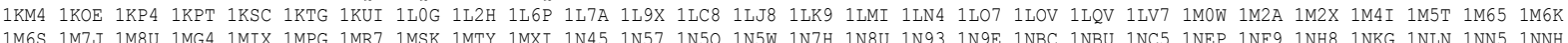
1NOG 1NPY 1NOD 1MG4 1MIX 1MPG 1NR 1NS 1NG

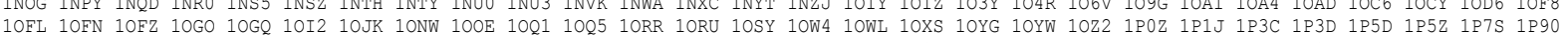

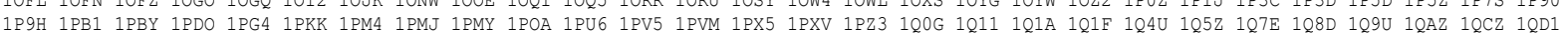

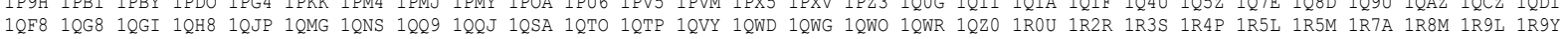

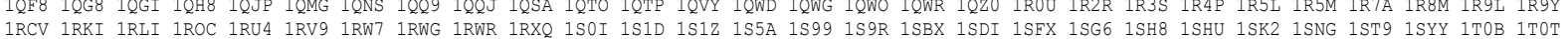

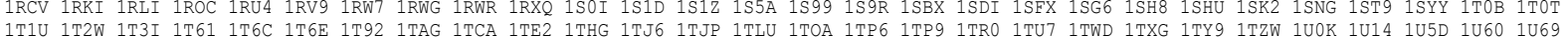

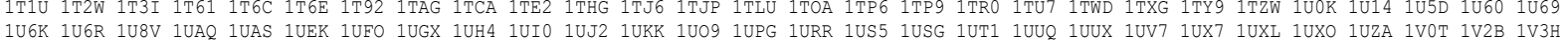

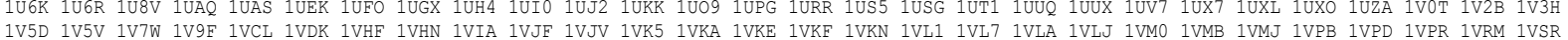

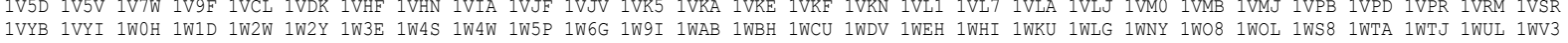

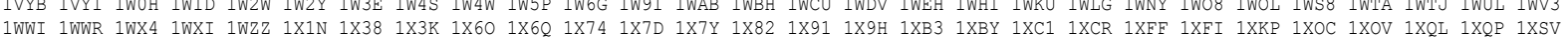

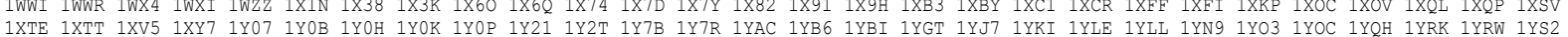

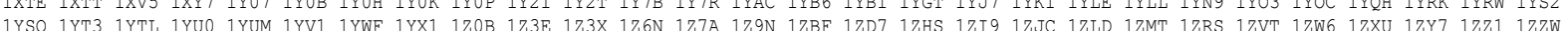
2A0B 2A14 2A2K 2A32 2A35 2A3M 2A4D 2A4O 2A5L 2A65 2A6B 2A6V 2A7B 2A94 2A9D 2A9I 2A9S 2AAL 2ABW 2AD6 2AEX 2AFW 2AG4 2AH5 2AHF 2AII 2AJ6 2AJ7 2AL1 2AML 2APJ 2AR1 2ARC 2ASF 2ATF 2AUO 2AVD 2AXC 2AXQ 2BOP 2BOV 2B2H 2B3L 2B4V 2B5H 2B5W 2B61 2B65 2B7U 2B8M 2BBA 2BDR 2BEK 2BEM 2BHY 2BJF

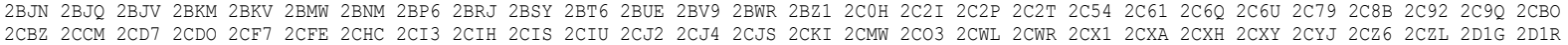
2D29 2D4P 2D5B 2D81 2DBY 2DC3 2DE3 2DEJ 2DE8 2DFY 2DG1 2DG5 2DQA 2DRI 2DSK 2DTC 2DTJ 2DUR 2DVM 2DVN 2DVT 2DWU 2DXA 2DY1 2E0P 2E11 2E1N 2E20 2E3R 2E7A 2E83 2E8G 2EAE 2EB6 2EB9 2EFE 2EG6 2EHG 2EI1 2EIE 2EJO 2EJN 2ELC 2ELF 2EPN 2ERB 2ET1 2ETJ 2ETV 2EV1 2EVR 2EWR 2EXO 2EX4 2EZ9 2F0C 2F1F 2F1K 2F1N 2F24 2F2B 2F4Q 2F57 2F5X 2F62 2F6D 2F6R 2F7V 2F8A 2F9F 2F9H 2FA1 2FC3 2FCT 2FDV 2FEX 2FGR 2FH1 2FHP 2FI9 2FLU 2FNU 2FOM 2FOZ 2FP1 2FQX 2FR2 2FSR 2FSU 2FSX 2FTN 2FUL 2FUR 2FWO 2FY7 2FYF 2FYG 2FZB 2GOW 2G1U 2G2C 2G40 2G50 2G62 2G82 2G8S 2GA8 2GAI 2GEY 2GFF 2GHS 2GI3 2GIB 2GIY 2GJ4 2GKJ 2GMQ 2GNP 2GS5 2GS8 2GU3 2GUI 2GUY 2GVK 2GWG 2GWM 2GX5 2GZ4 2H1C 2H1T 2H2R 2H6F 2H7J 2H7M 2H8G 2H98 2HA8 2HE2 2HEG 2HHC 2HHJ 2HHP 2HJE 2HKV 2HLY 2HMJ 2HNG 2HPO 2HPL 2HPS 2HQS 2HQY 2HSJ 2HUH 2HXO 2HX5 2HXW 2HY5 2HYT 2HZK 2I3D 2I3H 2I48 2I53 2I5I 2I5R 2I6H 2I74 2I7A 2I7G 2I8D 2I8E 2I9W 2IA1 2IBA 2IBD 2ICA 2ICG 2ICU 2IDL 2IEQ 2IF6 2IG7 2IGI 2IGP 2IIH 2IJA 2IK9 2IKB 2IKK 2IM9 2IMD 2IMH 2IMJ 2IML 2IMZ

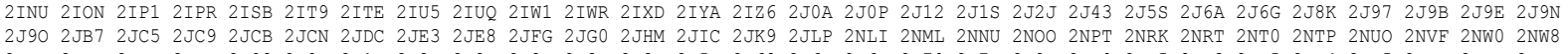
2NXF 2NXW 2NYH 2NYI 2002 200M 2010 202I 202P 202X 203F 203S 205V 2062 206P 206Y 2070 207I 2080 20A9 20B5 20C3 20C5 20D4 20D5 20EB 20FK 20IT

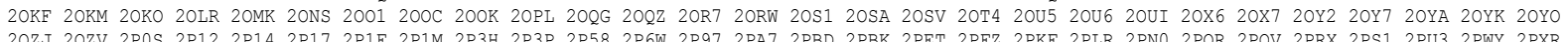
2OZJ 2OZV 2POS 2P12 2P14 2P17 2P1F 2P1M 2P3H 2P3P $2 \mathrm{P} 58$ 2P6W 2P97 2PA7 2PBD 2PBK 2PET 2PFZ 2PKF 2PLR 2PNO 2POR 2PQV 2PRX 2PS1 2PU3 2PWY 2PXR 2QIS 2QK1 2QKP 2QL8 2QLW 2QML 2QNI 2QNK 2QNL 2QRU 2QSA 2QSI 2QSW 2QUB 2QUD 2QUO 2QVU 2QW5 2QWU 2QXF 2QZC 2QRT 2QZU 2R1I 2R2C 2R4I 2R4Q 2R78 2R7G 2R85 2R8Q 2R9F 2RBD 2RBG 2RBW 2RCI 2RDC 2RDG 2RDS 2RFG 2RFQ 2RH3 2RIQ 2RJ2 2RKQ 2RKV 2RLD 2SAK 2SGA 2UUR 2UVJ 2UVK 2UVP 2UWA 2UY2 2UYK 2UYQ 2UYT 2UZ1 2VOH 2V2B 2V2G 2V38 2V3Z 2V4V 2V4X 2V5J 2V6A 2V6U 2V75 2V76 2V7K 2VAC 2VAP 2VB9 2VBP 2VBU 2VCL 2VFK 2VFO 2VFT 2VGO 2VG8 2VK6 2VLQ 2VLZ 2VMH 2VOW 2VPN 2VQ2 2VQP 2VRS 2VSM 2VUW 2VV6 2VVE 2VVP 2VXZ 2VY7 2VYW 2WOB 2W0I 2W31 2W3E 2 W3P 2 2W3X 2W52 2W5T 2W61 2W6K 2W7Z 2W86

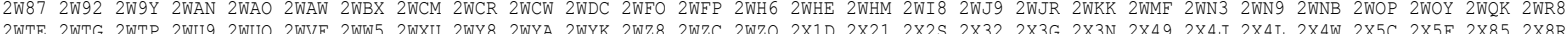
2WTE 2WTG 2WTP 2WU9 2WUQ 2WVF 2WW5 2WXU 2WY8 2WYA 2WYK 2WZ8 2WZC 2WZO 2X1D 2X21 2X2S 2X32 2X3G 2 2X3N 2 2X49 2 2X4J 2 2X4L 2 2X4W 2 2X5C 2 2X5F 2 2X85 2 2X8R

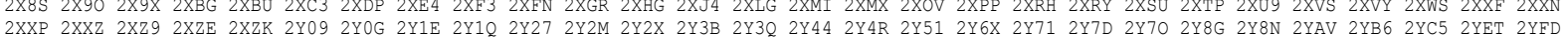

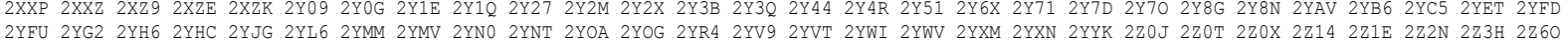

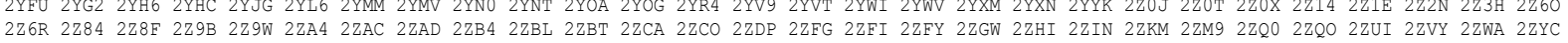

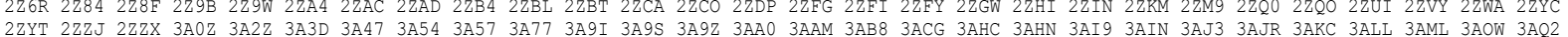
3ARQ 3ASQ 3ATS 3ATV 3AV3 3AWM 3AXD 3B2X 3B49 3B53 3B5E 3B6E 3B7C 3B7I 3B9T 3BA3 3BBD 3BB7 3BB9 3BCW 3BCY 3BEX 3BEM 3BFP 3BGY 3BHD 3BI1 3BI7

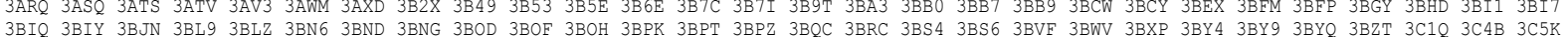
3BIQ 3BIY 3BJN 3BL9 3BLZ 3BN6 3BND 3BNG 3BOD 3BOF 3BOH 3BPK 3BPT 3BPZ 3BQC 3BRC 3BS4 3BS6 3BVF 3BWV 3BXP 3BY4 3BY9 3BYQ 3BZT 3C10 3C4B 3C5K

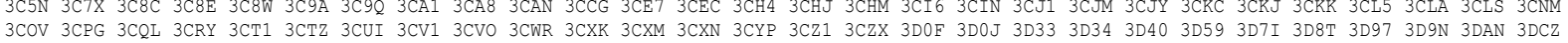

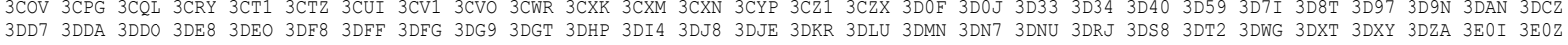
3E11 3E2V 3E3U 3E4W 3E7H 3E9K 3E9T 3EBV 3EC4 3EC9 3ECD 3EDF 3EDN 3EEA 3EF2 3EF3 3EF8 3EFY 3EH1 3EHG 3EJF 3EJN 3EK3 3EKI 3EKL 3ELG 3EMI 3EN0

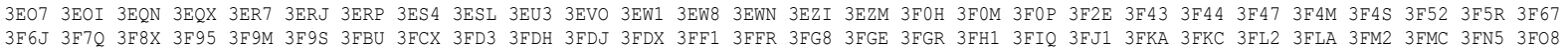
3FOJ 3FOT 3FP5 3FPF 3FPK 3FPW 3FRQ 3FRR 3FSU 3FT1 3FUN 3FUW 3FVS 3FW2 3FW8 3FWY 3FWZ 3FYB 3G02 3GOM 3G0T 3G3H 3G48 3G7U 3G7X 3G89 3GAE 3GAX 3GBY 3GDO 3GF3 3GF6 3GFP 3GG7 3GK6 3GKJ 3GKM 3GKR 3GMF 3GMG 3GMV 3GN6 3GNL 3GO9 3GOC 3GOQ 3GRS 3GS9 3GT3 3GVO 3GWI 3GWN 3GY9 3GYK 3GZA 3H05 3H09 3H0U 3H2S 3H3L 3H46 3H4O 3H4W 3H4Y 3H51 3H5L 3H68 3H6J 3H6Q 3H7C 3H7H 3H7I 3H7V 3H8G 3H8T 3H93 3H9B 3H9M 3HA2 3HAP 3HBM 3HD4 3HDO 3HDX 3HID 3HJ4 3HJB 3HJR 3HKW 3HLO 3HLZ 3HMZ 3HNO 3HN5 3HNA 3HNH 3HO6 3HOI 3HP7 3HQ1 3HQM 3HQX 3HRP 3HSY 3HTN 3HU5 3HV2 3 HVV 3 HYQ 3 HZ6 3 I07 3 IOY 3I0Z 3I1A 3I24 3I47 3I48 3I4Z 3I6I 3I96 3IAR 3IBZ 3IDU 3IEE 3IGS 3IHV 3IJM 3IKB 3ILF 3ILR 3ILS 3ILW 3IM0 3IM1 3IOX 3IQ1 3IRB 3IRP 3IRS 3ISQ 3IT3 3IU5 3IU6 3IUO 3IV4 3IVZ 3JRV 3JS8 3JSC 3JSY 3JUO 3JXS 3JYZ 3JZ9 3K00 3K0B 3KOX 3K1U 3K1W 3K1Z 3K26 3K3C 3K40 3K4I 3K6M 3K6Q 3K7G 3K8 3KB9 3KBG 3KBY 3KDW 3KEO 3KGO 3KG9 3KGW 3KGY 3KI6 3KIZ 3KLO 3KMH 3KMT 3KMV 3KOR 3KQR 3KS6 3KSN 3KSX 3KT7 3KU3 3KV1 3KVH 3KVS 3KWS 3L00 3L1F 3L1W 3L34 3L3X 3L40 3L4A 3L4E 3L4H 3L4N 3L51 3L6B 3L8Q 3LAT 3LB4 3LCC 3LD7 3LEW 3LFJ 3LFR 3LFU 3LGB 3LGI 3LHE 3LHO 3LI3 3LID 3LIM 3LIO 3LKM 3LLO 3LLP 3LLX 3LM2 3LOG 3LOP 3LRT 3LSC 3LTJ 3LVU 3LXO 3LX3 3LXS 3LY1 3LYG 3LYH 3LZA 3LZN 3LZW 3MOH 3M0J 3M1H 3M1T 3M1U 3M66 3M6L 3M76 3M70 3M84 3M86 3M92 3MAK 3MD9 3MDM 3MDQ 3ME7 3MF7 3MHZ 3MIL 3MK1 3MKO 3ML1 3MMG 3MOK 3MPC 3MT0 3MVU 3MW8 3MWL 3MWZ 3MXN 3MXO 3MXU 3MXZ 3MYV 3MZ2 3MZF 3MZQ 3NO3 3NOU 3NOX 3N1E 3N34 3N37 3N6Y 3N79 3N80 3N8I 3N9C 3N9K 3NA5 3NAD 3ND1 3NDD 3NEC 3NEH 3NEU 3NFT 3NEW 3NGV 3NJC 3NJK 3NRQ 3NM6 302 30483040 30AM 3OBE 30BU 30C9 30CC 30CV 30EB 30EP 30F5 30GH 30HG 30IO 30JC 30KX 30LL 30M1 30MC 30MD 30N1 30N9 30NH 30NO 3008 3000 300S 3OOU 3OQI 3OS4 3OS7 3OSE 3OST 3OSX 30T9 3OTI 3OTM 3OU2 3OUG 3OUI 3OUL 30XH 3P02 3P1G 3P2C 3P2E 3P2T 3P3G 3P3V 3P4G 3P97 3P9A 3P9V 3PA6 3PBT 3PCX 3PCZ 3PDD 3PE7 3PFE 3PFT 3PG6 3PGU 3PI6 3PI7 3PJO 3PJP 3PJY 3PLN 3PM2 3PMD 3PME 3PMS 3PNA 3PO8 3POF 3POH 3POP 3POW 3PPM 3PR6 3PU9 3PUI

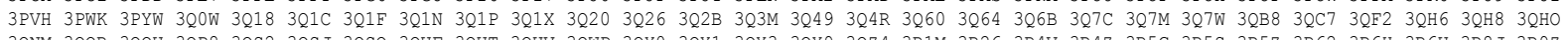
3QNM 3OOR 3QOU 3OP8 3OS2 3OSJ 3OSO 3OUF 3OUT 3OUV 3OWP 3OYO 3OY1 3OY3 3OY9 3OZ4 3R1M 3R26 3R4V 3R4Z 3R5G 3R5S 3R5Z 3R62 3R6H 3R6U 3R8J 3R9Z

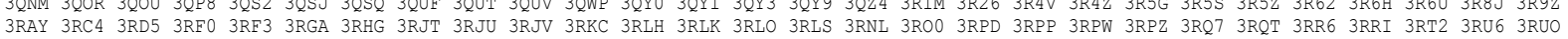

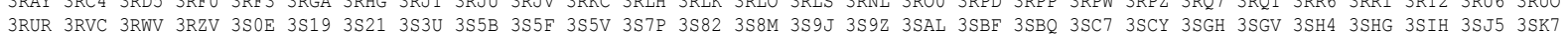

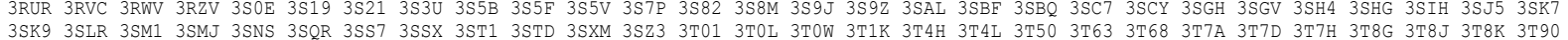

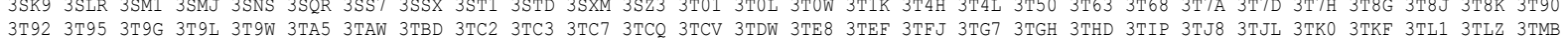

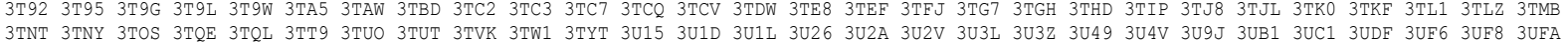

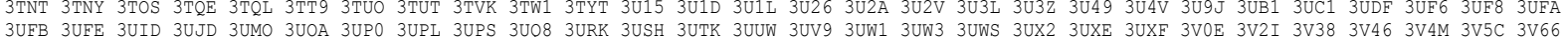

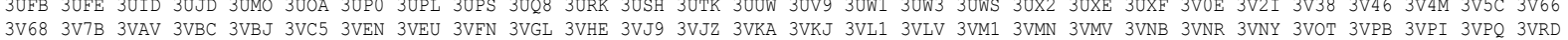

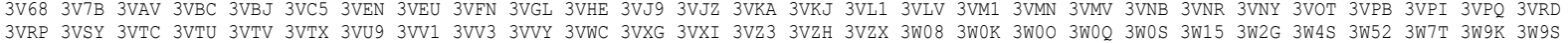
3VRP 3VSY 3VTC 3VTU 3VTV 3VTX 3VU9 3VI 3VV3 3VVY 3VWC 3VXG 3VXI 3VZ3 3VZH 3VZX 3W08 3WOK 3W00 3W0Q 3W0S 3W15 3W2G 3W4S 3W52 3W7T 3W9K 3W9S 3WA7 3ZBO 3ZFN 3ZHF 3ZJH 3ZJI 3ZJL 3ZJM 3ZN6 3ZQI 3ZQK 3ZRO 3ZRI 3ZRV 3ZSU 3ZT9 3ZUI 3ZUZ 3ZWF 3ZWT 3ZX4 3ZYH 3ZYL 3ZYP 3ZYU 4A0D 4A2B 4A30

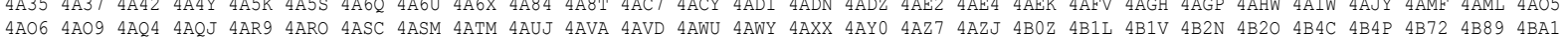

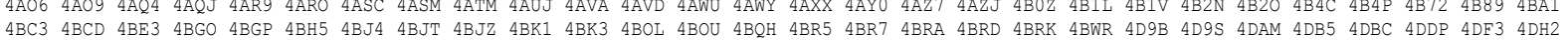
4DKK 4DL8 4DLN 4DM5 4DMC 4DMI 4DMK 4DMV 4DN7 4DNX 4DNY 4DO7 4DOI 4DOM 4DOY 4DQ6 4DQA 4DQD 4DRR 4DTH 4DUH 4DV8 4DWD 4DWN 4DY5 4DYQ 4DZO 4E15 4E1A 4E1B 4E2U 4E32 4E4T 4E5V 4E70 4EAD 4EBJ 4EBY 4ECF 4ECP 4EE7 4EFO 4EF8 4EHS 4EHU 4EKX 4EL6 4EMT 4EOO 4EPZ 4EQB $4 E Q G$ 4EQL 4ES5 4ES8 4ESQ 4ESU 4ESW 4EU3 4EUS 4EW1 4EW7 4EWR 4EX7 4EYS 4FOJ 4F1J 4F3N 4F54 4F67 4F6T 4F8L 4FBS 4FCJ 4FCS 4FDB 4FDM 4FDV 4FE3 4FFL 4FGQ 4FKZ 4FNV 4FO7 4FOJ 4FP1 4FQS 4FR7 4FUS 4FVC 4FVG 4FVY 4FX5 4FYP 4G1J 4G1R 4G22 4G26 4G29 4G38 4G3V 4G48 4G4K 4G4P 4G4X 4G54 4G55 4G79 4G9M 4G9P 4G90 4GB5 4GBM 4GCY 4GD6 4GE3 4GEI 4GEK 4GFO 4GET 4GGJ 4GH9 4GHG 4GHI 4GHN 4GKB 4GLQ 4GMM 4GNB 4GNF 4GNM 4GNO 4GNV 4GNY 4GOB $4 \mathrm{GPV}$ 4GQ6 4GT6 4GTA 4GUJ 4GVR 4GWK 4GXB 4GYF 4GYW 4GZJ 4GZK 4HO4 4H08 4HOC 4H14 4H2G 4H41 4H59 4H5G 4H5S 4H6R 4H7U 4H7Y 4H8N 4HBA 4HBZ 4HD9 4HDK 4HE7 4HFS 4HGI 4HH3

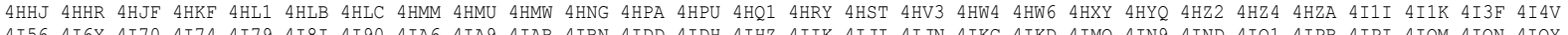
4I56 4I6X 4I70 4I74 4I79 4I8I 4I90 4IA6 4IA9 4IAB 4IBN 4IDD 4IDH 4IHZ 4IIK 4IJI 4IJN 4IKC 4IKD 4IMQ 4IN9 4IND 4IO1 4IPB 4IPI 4IQM 4IQN 4IQY

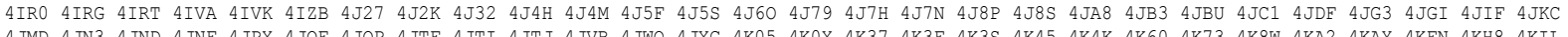

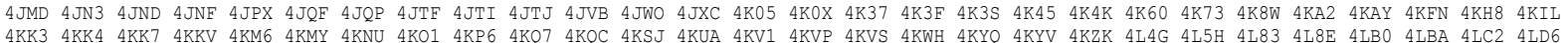
4LFG 4LFY 4LG1 4LHR 4LLE 4LLS 4LMI 4LQB 4LRD 4LRU 4LSM 4LUI 4LW2 5CYT 5NUL 7ODC 


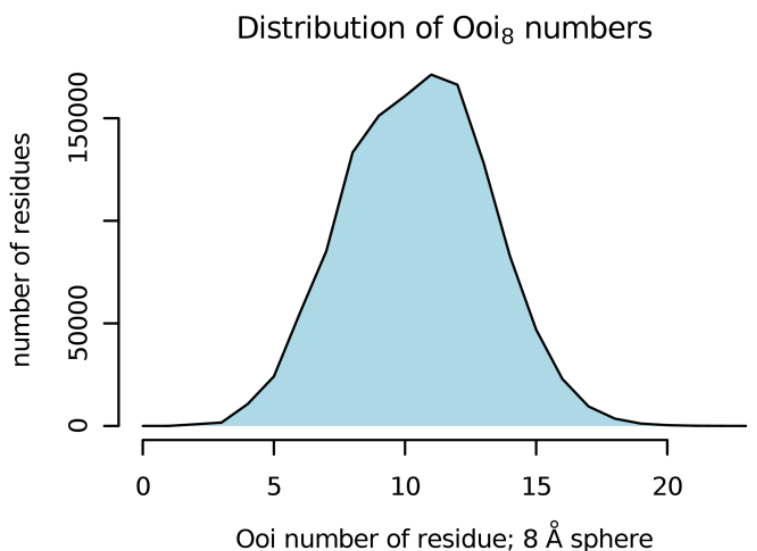

Distribution of $\mathrm{ACN}_{8}$ numbers

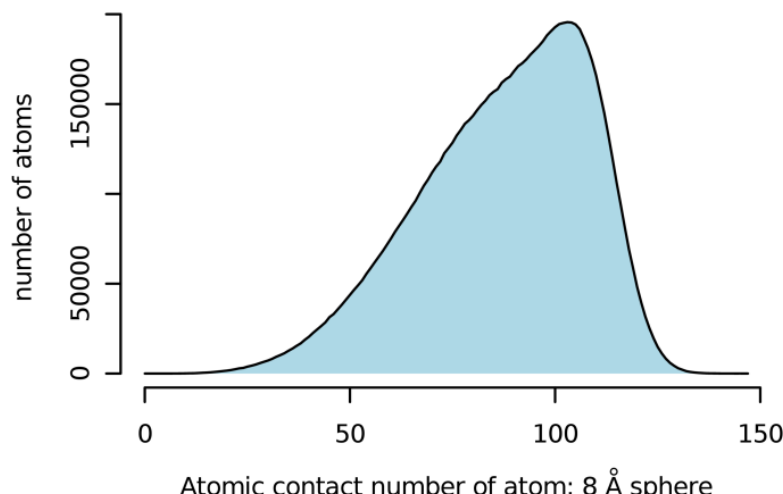

Atomic contact number of atom; $8 \AA$ sphere
Distribution of $\mathrm{Ooi}_{14}$ numbers

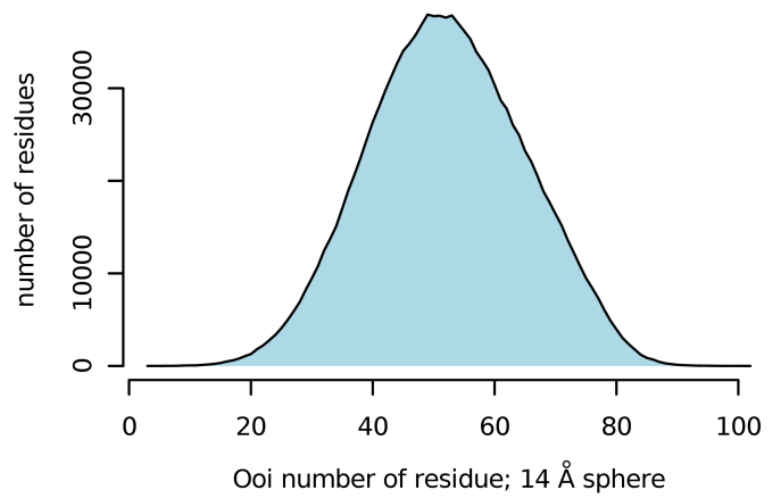

Distribution of $\mathrm{ACN}_{14}$ numbers

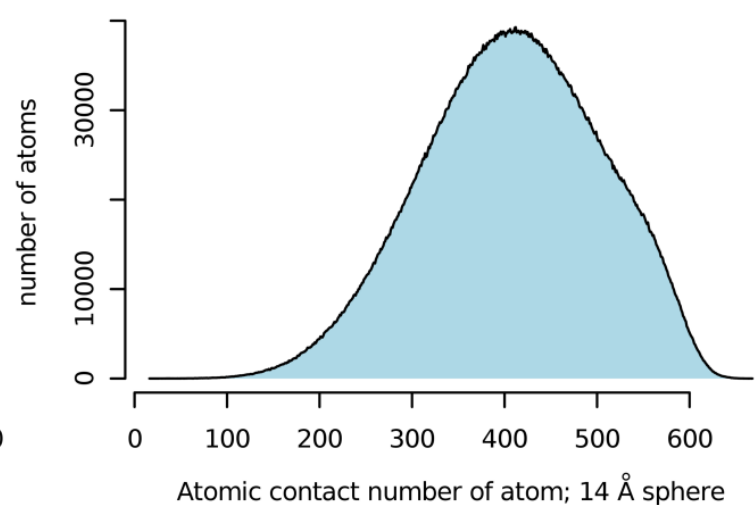

Figure S1 Distribution of Ooi numbers and atomic contact numbers (ACN) for radii of $8 \AA$ and $14 \AA$ for the 2,704 selected PDB structures. Note that both Ooi numbers and ACNs are discrete metrics; they can only take integer values. 
1. Let the variable $a$ denote a single atom.

2. Let the set $A$ describe the set of all non-hydrogen protein atoms within the same PDB structure.

3. Choose a packing density function $P: A \rightarrow \mathbb{R}$ mapping every atom to a packing density. For this paper $P$ was defined using atomic contact numbers with a range of $14 \AA$.

4. Let $B: A \rightarrow \mathbb{R}$ map every atom to its atomic $\mathrm{B}$ factor (atomic displacement parameter).

5. The function of average B factors returns the arithmetic mean of the atomic B factors of a set of atoms $X \subseteq A$ :

$\bar{B}(X):=\frac{\sum_{a \in X} B(a)}{|X|}$

6. Define the packing density environments $E_{n}:=\{a \in A \mid P(a)=n\}$ as sets, that contain all atoms with the packing density $n$ as determined by $P$. The set of all nonempty $E_{n}$ is a partition of $A$.

7. Similar packing density environments $S_{m}$ are defined as sets of all atoms with a packing density similar to $m \in \mathbb{R}$ :

$$
S_{m}:=\bigcup_{n \in \operatorname{sim}(m)} E_{n}
$$

8. Similarity can be defined by range, e.g. $\operatorname{sim}(m):=\{x \in \mathbb{R} \mid m-\varepsilon \leq x \leq m+\varepsilon\}, \varepsilon \geq 0$ or by binning, e.g. $\operatorname{sim}(m):=\left\{x \in \mathbb{R} \mid\left\lfloor\frac{x}{z}\right\rfloor=\left\lfloor\frac{m}{z}\right\rfloor\right\}$ with a bin size $z>0$. Here the latter definition was used with $z=10$.

9. Define $\mathrm{B}_{\text {Damage }}$ of an atom $a$ as the atomic $\mathrm{B}$ factor divided by the average $\mathrm{B}$ factor of all nonhydrogen protein atoms within the same PDB structure and with a similar packing density as $a$ :

$$
B_{\text {Damage }}(a):=\frac{B(a)}{\bar{B}\left(S_{P(a)}\right)}
$$

Figure S2 Mathematical description of $B_{\text {Damage }}$ calculation. 


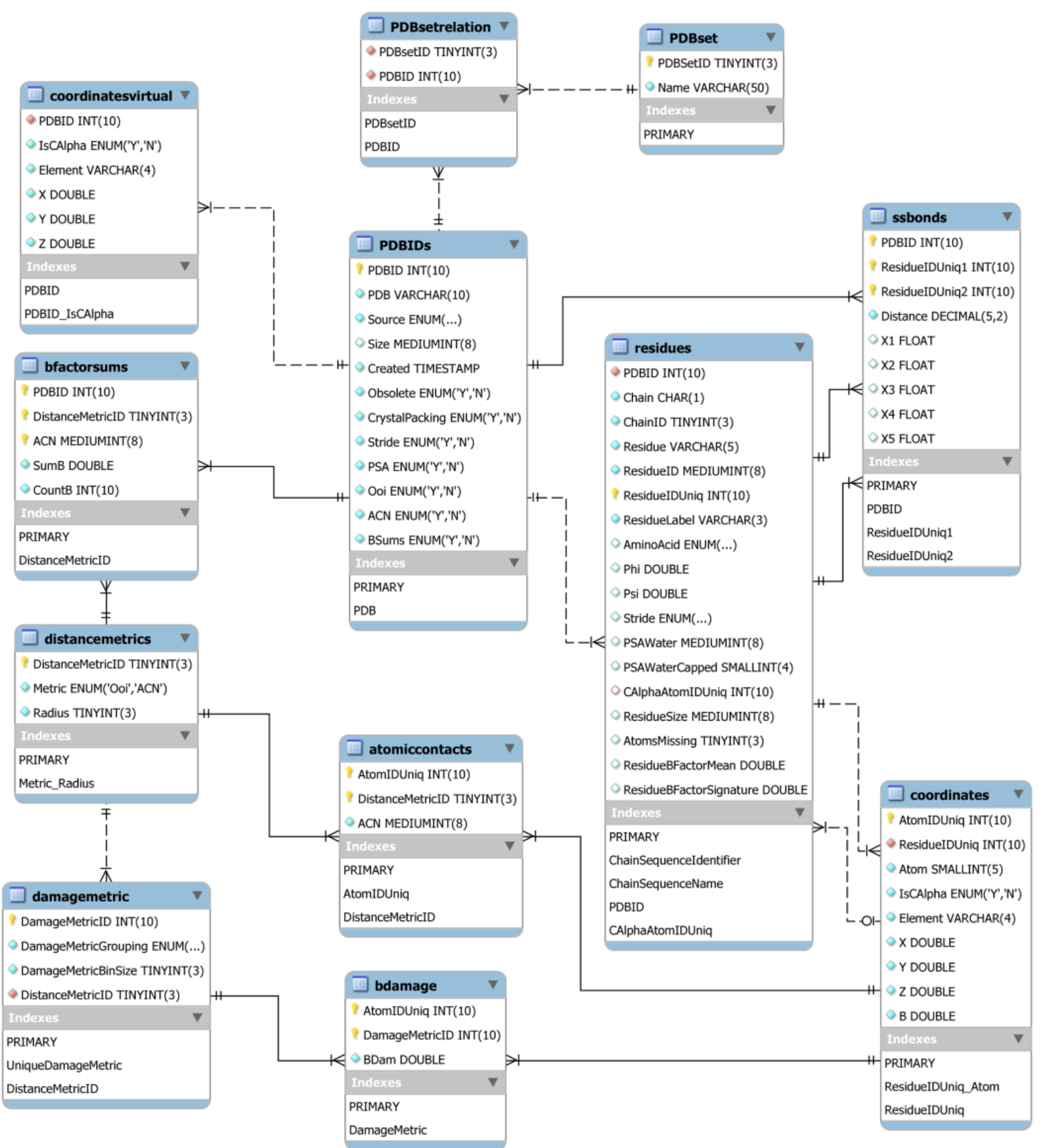

Figure S3 Entity-Relationship diagram of the relational database used for this investigation.

Diagram prepared using MySQL Workbench 6.0 (http://mysqlworkbench.org/). 


\section{ANODE peaks vs $B_{\text {Damage }}$}

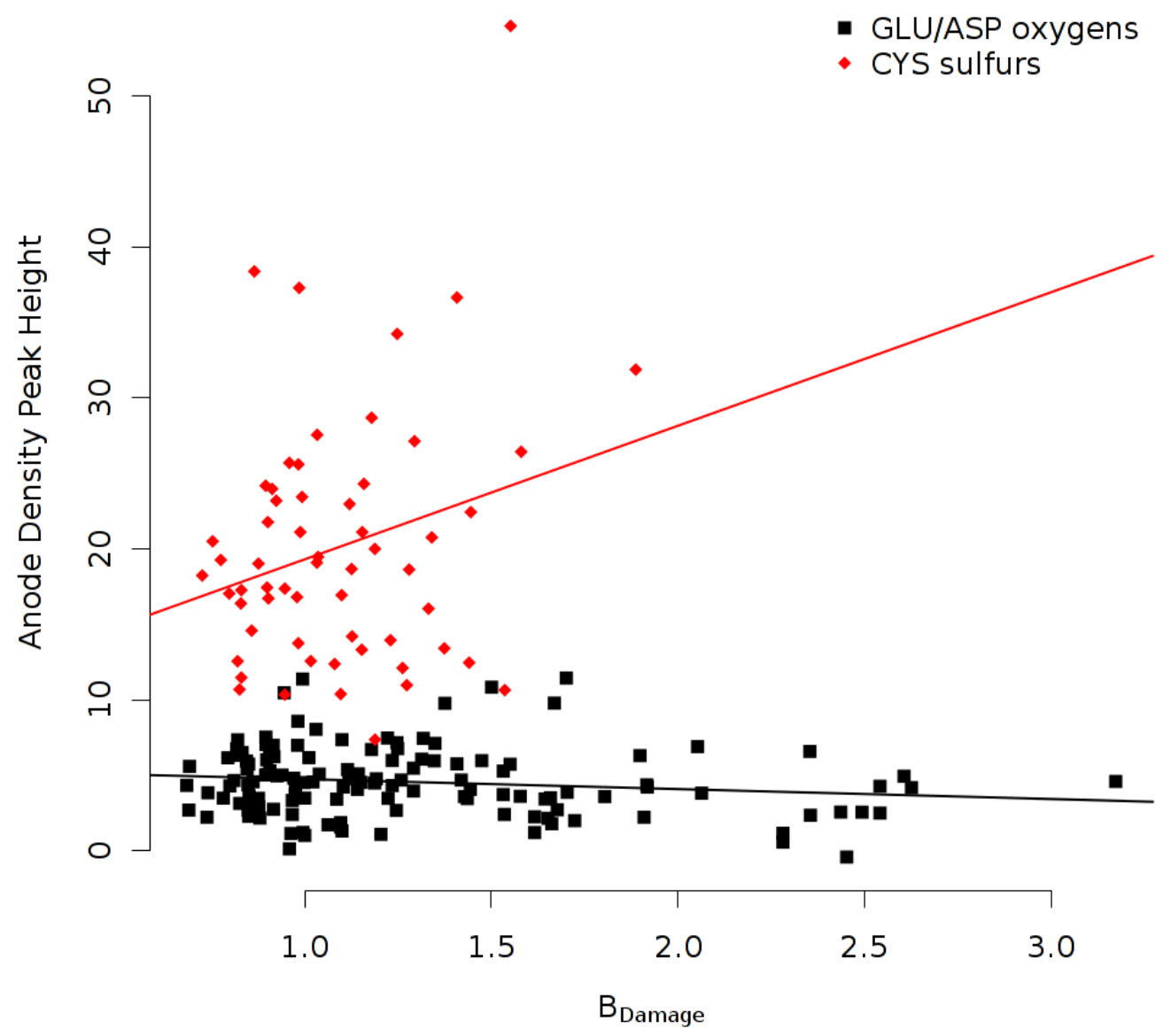

Figure S4 Peak heights of electron density difference maps around the cysteine sulfurs (red) and the terminal oxygens of glutamic and aspartic acids (black) plotted against their $B_{\text {Damage }}$ values for the six Nanao protein datasets. Lines show least squares fits to the points. Peak heights were calculated using SHELXC (Sheldrick, 2010) and ANODE (Thorn \& Sheldrick, 2011). $B_{\text {Damage }}$ values were calculated for the relevant high-dose dataset.

There is some evidence for a correlation of high $B_{\text {Damage }}$ values and strong electron density peak differences for cysteine sulfurs $(\mathrm{CC}=0.25, \mathrm{p}=0.05)$. The apparent inverse correlation for GLU/ASP oxygens is not statistically significant $(\mathrm{CC}=-0.15, \mathrm{p}=0.09)$. 


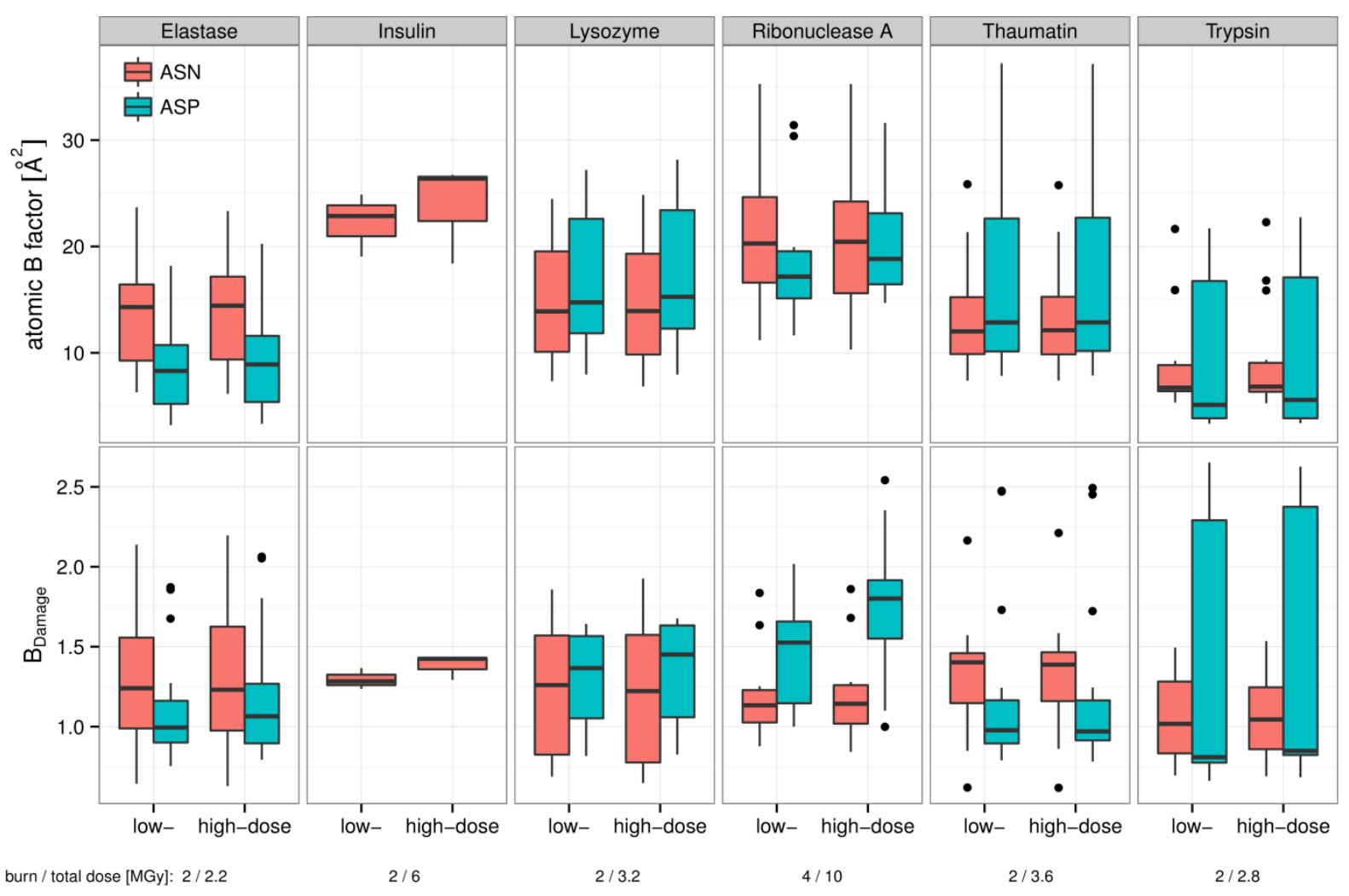

Figure S5 $B$-factor (above) and $B_{\text {Damage }}$ (below) of ASP and ASN O $\delta$ atoms of the six Nanao et al. (2005) proteins in two different dose states. The deterioration of the ASP side chain termini of ribonuclease A cannot be observed from the $B$-factor alone, but is clearly indicated by $B_{\text {Damage. }}$ The ASN oxygens remain unaffected.

\section{References}

Sheldrick, G. M. (2010). Acta Cryst. D66, 479-485.

Thorn, A. \& Sheldrick, G. M. (2011). J. Appl. Cryst. 44, 1285-1287. 